\title{
Patient Satisfaction With Hospital Care Provided by Hospitalists and Primary Care Physicians
}

\author{
Adrianne Seiler, MD ${ }^{1,2,3^{*}}$, Paul Visintainer, $\mathrm{PhD}^{4}$, Richard Brzostek, MA ${ }^{5}$, Michael Ehresman, MBA ${ }^{6}$, \\ Evan Benjamin, $\mathrm{MD}^{1,3,7}$, Winthrop Whitcomb, MD ${ }^{1,2,3,7}$, Michael B. Rothberg, MD, $\mathrm{MPH}^{3,7,8}$
}

\begin{abstract}
${ }^{1}$ Division of Healthcare Quality, Baystate Medical Center, Springfield, Massachusetts; '2Division of Hospital Medicine, Baystate Medical Center, Springfield, Massachusetts; ${ }^{3}$ Division of General Medicine, Tufts University School of Medicine, Boston, Massachusetts; ${ }^{4}$ Division of Academic Affairs, Baystate Medical Center, Springfield, Massachusetts; ${ }^{5}$ Division of Customer Satisfaction and Market Research, Baystate Medical Center, Springfield, Massachusetts; 'Division of Decision Support, Baystate Medical Center, Springfield, Massachusetts; 'Center for Quality of Care Research, Baystate Medical Center, Springfield, Massachusetts; ' Division of General Medicine and Geriatrics, Baystate Medical Center, Springfield, Massachusetts.
\end{abstract}

BACKGROUND: Compared to hospital care provided by primary care physicians (PCPs), the hospitalist model provides equal-to-superior efficiency and outcomes; however, little is known about how the model affects patient satisfaction.

METHODS: Random patient satisfaction telephone interviews were conducted on discharged adult medicine inpatients at 3 Massachusetts hospitals between 2003 and 2009. Questionnaires included variables assessing patient satisfaction with various physician care domains. Patient age, gender, admission year, education level, language, illness severity, emergency room admission status, institution, and attending physician type were extracted from billing records. We used adjusted multivariable models to compare patient satisfaction with hospitalists and PCPs for domains of: physician care quality, physician behavior, pain management, communication.

RESULTS: Inpatients completed discharge surveys for 8295 encounters (3597 hospitalist, 4698 PCP). Multivariate- adjusted satisfaction scores for physician care quality were slightly higher for PCPs than hospitalists (4.24 vs $4.20, P=$ $0.04)$; there was no statistical difference at any individual hospital, and no difference among different hospitalist groups. Patient ratings of hospitalists and PCPs for behavior, pain control, and communication were equivalent (all $P$ values $>0.23$ ). In multivariable models, hospitalists and PCPs had similar adjusted proportions in the highest satisfaction category $(79.2 \%$ vs $80.5 \%$, respectively, $P=$ 0.17 ) and lowest category (5.1\% vs $4.5 \%$, respectively, $P=$ $0.19)$. Quality ratings of both groups improved equivalently $(P$ slope interaction $=0.47)$ but significantly over time $(\mathrm{PCP}$ 4.21 (2003) to 4.36 (2009), hospitalist 4.11 to $4.33, P \Delta$ $<0.001)$.

CONCLUSIONS: Patients appear similarly satisfied with inpatient care provided by several hospitalist models and by primary care physicians. Journal of Hospital Medicine 2012;7:131-136. (c) 2011 Society of Hospital Medicine
Over the past decade, hospital medicine has been the nation's fastest-growing medical specialty. According to the American Hospital Association's (AHA) 2009 survey, $58 \%$ of United States (US) hospitals now have hospital medicine programs, and for hospitals with 200 or more beds, this figure is $89 \% .^{1}$ In 2009 , the AHA estimated that the number of US hospitalists would increase to over 34,000 by 2011 , over double that of the 16,000 present in $2005 .^{1}$ Studies demonstrate that, compared to a system where primary care physicians provide inpatient care, the hospitalist model improves efficiency while maintaining at least equal patient outcomes. ${ }^{2-11}$ However, scant data exist as to the effects of hospitalists on patient satisfac-

*Address for correspondence and reprint requests: Adrianne Seiler, MD, Division of Hospital Medicine, Baystate Medical Center, 759 Chestnut St, Springfield, MA 01199; Tel.: 413-794-4320; Fax: 413-794-1767; E-mail: Adrianne.Seiler@bhs.org

Additional Supporting Information may be found in the online version of this article.

Received: March 22, 2011; Revised: August 4, 2011; Accepted: August 13, 2011

2011 Society of Hospital Medicine DOI 10.1002/jhm.973

Published online in Wiley Online Library (Wileyonlinelibrary.com). tion. ${ }^{12}$ Understanding how care models affect patient experience is vital in the current environment of healthcare reform and performance reporting, especially in light of the Centers for Medicare and Medicaid Services' (CMS) efforts to link the patient experience to reimbursement through "value-based" purchasing. ${ }^{13}$ Value-based purchasing is a strategy to encourage and reward excellence in healthcare delivery through differential reimbursement based on defined performance measures. As one part of valuebased purchasing, hospital reimbursement will be linked to patient-experience measures, including patient ratings of their doctor's ability to communicate with them and other questions assessing patient satisfaction with their hospital stay. ${ }^{14}$

In the outpatient setting, trust is the variable most strongly associated with patient satisfaction. ${ }^{15-18}$ In contrast to PCPs, who may develop relationships with patients over years, hospitalists often first meet a patient in the hospital and must engender trust quickly. In addition, hospitalists work in shifts and may not be responsible for the same patients each day. Since continuity is positively related to trust, ${ }^{19,20}$ there is reason to believe satisfaction with hospitalist 
care might be lower than satisfaction with care provided by PCPs. We report on 8295 patients and 6 years experience with hospitalist programs at 3 hospitals. Based on the known link between continuity and patient satisfaction, we hypothesized that patient satisfaction would be lower with hospitalists than with primary care internists.

\section{METHODS}

\section{Setting}

Our study was conducted at 3 Western Massachusetts hospitals affiliated with Baystate Health, an integrated healthcare delivery system. These included 2 small community hospitals $(<100$ beds) and a 653-bed tertiary care, academic teaching hospital. Hospitalist services were established at the tertiary care center in 2001 and at the community hospitals in 2004 and 2005; the programs have evolved over time. In addition, the tertiary care center has 3 different hospitalist groups: an academic group that is employed by the hospital and works with house staff, a hospitalist service that is owned by the hospital and cares for patients from specific outpatient practices, and one that is privately owned caring for patients from another group of practices. The community hospitals each have a single, hospital-owned service. Primary care physicians also provide inpatient care at all 3 institutions, although their number has decreased over time as the hospitalist programs have grown. All hospitalist services varied in the number of consecutive days in a rounding cycle (degree of continuity), and which services had an admitting team (single initial physician encounter with a different rounding physician) versus a single physician being both the admitting and rounding physician. Consequently, continuity, as measured by the number of different physicians caring for an individual patient during 1 hospitalization, would be expected to vary depending on the type of hospitalist service and the length of stay. Likewise, patients admitted by their primary care physician's office may have been cared for by either their PCP or a practice colleague. All hospitalists and PCPs care for inpatients having similar hospital experiences, as all aspects of a patient's care (including the medical wards, nursing staff, discharge planners, and information systems) are identical, regardless of physician designation. The study was approved by Baystate Health System's Institutional Review Board.

\section{Data Collection}

Since February 2001, Baystate Health, in conjunction with Professional Research Consultants, Inc (PRC), has conducted scripted postdischarge patient satisfaction telephone interviews of random discharged adult medicine patients, with Hospital Consumer Assessment of Healthcare Providers and Systems (HCAHPS) questions added in January 2007. Approximately 50 surveys per quarter, per hospital floor, were conducted. Trained PRC staff assessed up to 115 variables encompassing the inpatient experience. We limited our analysis to those domains that reflected satisfaction with physician care, including satisfaction with physician care quality, physician communication, physician behavior, and pain management. The survey responses were scored, depending on question type, with: never, sometimes, usually, always (HCAHPS); or excellent, very good, good, fair, poor (PRC). Each score was converted to a numeric equivalent, with the highest score ( 4 or 5 , depending on scale used) being best and 1 being worst. The specific questions are included in Supporting Appendix A in the online version of this article.

Additional patient information for respondents was extracted from the hospitals' billing database, using medical record numbers, and included age, gender, admission year, education level, language, illness severity, emergency room (ER) admission status, institution, and attending physician type (academic hospitalist $[\mathrm{AH}]$, hospital-owned hospitalist $[\mathrm{HOH}]$, private hospitalist $[\mathrm{PH}]$, or primary care physician [PCP]). It was not possible to distinguish whether PCP patients were cared for by their own PCP or a colleague from the same practice.

\section{Statistical Analysis}

Patient satisfaction data were derived from survey responses of adult inpatients cared for by hospitalists or PCPs between January 1, 2003 and March 31, 2009. The primary outcome was patient-reported satisfaction with physician care quality measured on a 5point Likert scale. In a secondary analysis, physician groups were compared on the proportion of responses that were "excellent" (a score of " 5 " on the Likert scale) and the proportion that were "poor" (a score of " 1 "). Other secondary outcomes included patient satisfaction ratings of physician behavior, pain management, and communication. Averages and percent ranking "excellent" and "poor" were calculated for each hospitalist group and for PCPs. Other outcomes analyzed included average patient satisfaction with physician care quality, both over time and stratified by the presence or absence of having an established PCP prior to admission.

In view of the large sample size, Likert-scale responses were analyzed as continuous outcomes. For unadjusted comparisons among hospitalist groups, $t$ tests and 1-way ANOVAs were conducted for the scales scores, while chi-square tests were used for dichotomous outcomes. For multivariable analyses, multiple linear regression was used for continuous outcomes. For dichotomous outcomes, adjusted prevalence ratios were estimated using Poisson regression with robust standard errors. ${ }^{21}$ All multivariable models controlled for sex, marital status, illness severity, 
age group, ethnicity, length of stay, and emergency room admission. Observations with missing data were excluded from analyses. Differences in bivariable and multivariable analyses were considered significant at a critical test level of $5 \%$. Prevalence ratios are reported with 95\% confidence intervals. All analyses were conducted in Stata, version 11 (StataCorp, College Station, TX).

\section{RESULTS}

Of patients who were reached by telephone, $87 \%$ agreed to participate in the hospital survey. However, most patients could not be reached by phone; thus our estimated response rate, including those who could not be reached, was $27 \%$. For the subset of patients interviewed using the HCAHPS protocol, the response rate was $40 \%$. Our final sample included 8295 patients (3597 cared for by 59 hospitalists and 4698 by 288 PCPs) interviewed between 2003 and 2009. Three-

\begin{tabular}{llll}
\multicolumn{4}{l}{ TABLE 1. Characteristics of Patients Cared for by } \\
\multicolumn{4}{l}{ Hospitalists and Primary Care Physicians } \\
\hline & PCP & Hospitalist & \\
Characteristic & $\mathrm{N}=4698$ & $\mathrm{~N}=3597$ & $P$ Value \\
\hline Age (mean, SD) & $63.5(16.6)$ & $63.7(16.3)$ & 0.53 \\
Male sex (\%) & 44.9 & 46.2 & 0.28 \\
White race (\%) & 85.3 & 83.2 & 0.01 \\
Married (\%) & 49.1 & 48.7 & 0.69 \\
English spoken at home (\%) & 96.0 & 97.0 & 0.09 \\
At least some college education (\%) & 47.1 & 43.7 & 0.22 \\
Admitted through the emergency department (\%) & 84.3 & 92.5 & $<0.001$ \\
Average illness severity rating (mean, SD) & $2.0(0.8)$ & $2.2(0.8)$ & $<0.001$ \\
Average perceived health score (mean, SD) & $3.0(1.2)$ & $2.8(1.2)$ & 0.01 \\
Average length of stay (days) (mean, SD) & $4.0(3.6)$ & $4.3(4.3)$ & $<0.001$ \\
Discharged home (\%) & 87.9 & 88.5 & 0.73 \\
\hline
\end{tabular}

quarters of the patients were from the tertiary care center, whereas $17 \%$ and $8 \%$ were from each of the community hospitals (see Supporting Appendix B in the online version of this article). Patient characteristics appear in Table 1. Patients cared for by hospitalists were similar to those cared for by PCPs in terms of age, sex, marital status, education, and language, but hospitalist patients were more likely to have been admitted through the emergency department $(93 \%$ vs $84 \%, P<$ $0.001)$ and less likely to be white $(83 \%$ vs $85 \%, P=$ $0.01)$. Patients cared for by hospitalists also had higher average illness severity score $(2.2 \pm 0.8$ vs $2.0 \pm 0.8, P$ $<0.001)$, longer average $\operatorname{LOS}(4.3 \pm 4.3$ vs $4.0 \pm 3.6$, $P<0.001)$, and lower mean perceived health score $(2.8$ \pm 1.2 vs $3.0 \pm 1.2, P=0.01$ ).

Unadjusted patient reported satisfaction with physician care quality was slightly greater for PCPs than hospitalists (4.25 vs $4.19, P=0.009)$. After multivariable adjustment, the difference was attenuated but persisted (4.24 vs $4.20, P=0.04$ ). We found no statistical difference among the hospitals or the specific hospitalist groups in terms of satisfaction with overall physician care quality (Figure 1). There were no statistical differences in patient satisfaction ratings of hospitalist and PCPs for the subdomains of behavior, pain, and communication (Table 2). There were also no differences in the proportion of patients cared for by hospitalists or PCPs who rated their physicians in the highest satisfaction category $(79 \%$ vs $81 \%, P=$ $0.17)$ or the lowest ( $5 \%$ vs $5 \%, P=0.19)$. Among patients cared for by academic hospitalists, there was no difference in satisfaction rating between those patients who had a designated primary care physician in the outpatient setting and those who did not (4.22 \pm 0.94 vs $4.19 \pm 0.94, P=0.97)$. Finally,

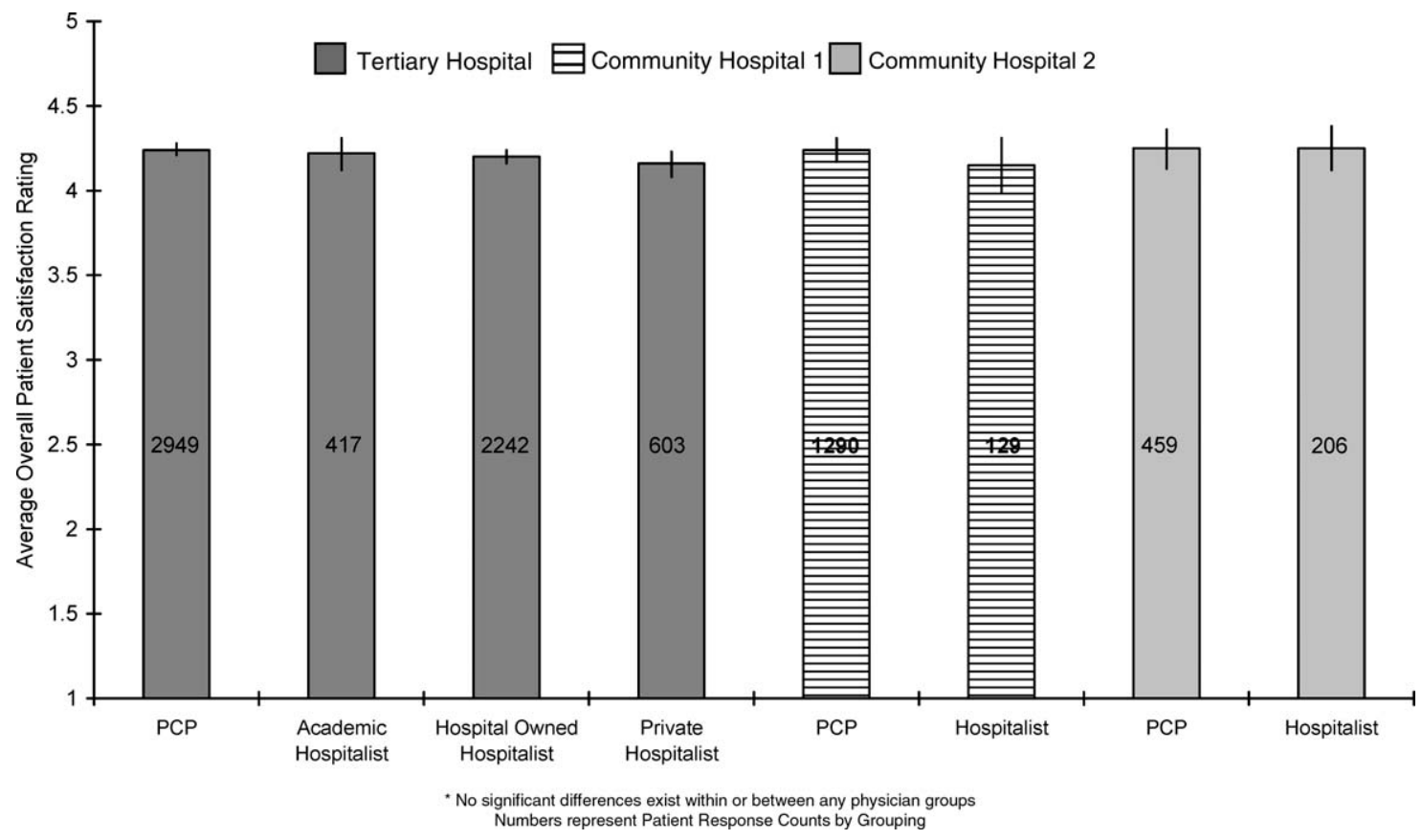

FIG. 1. Patient satisfaction with physician care quality, adjusted. Abbreviations: PCP, primary care physician. 
TABLE 2. Adjusted Average Patient Satisfaction With Physician Rating by Category

\begin{tabular}{|c|c|c|c|c|}
\hline & & PCP & Hospitalist & $P$ Value \\
\hline Satisfaction & Overall, you would rate the quality of doctor care as:* & $4.24[4.21,4.27]$ & $4.20[4.17,4.23]$ & 0.04 \\
\hline Behavior & Doctors treated you with courtesy/respect ${ }^{\dagger}$ & $3.77[3.73,3.82]$ & $3.78[3.73,3.82]$ & 0.88 \\
\hline \multirow[t]{2}{*}{ Pain control } & Pain management by hospital staff* & $4.11[4.08,4.14]$ & $4.09[4.05,4.12]$ & 0.35 \\
\hline & Staff did everything to help with pain ${ }^{\dagger}$ & $3.73[3.66,3.80]$ & $3.68[3.62,3.75]$ & 0.33 \\
\hline \multirow[t]{3}{*}{ Communication skills } & Doctors listened carefully to you ${ }^{\dagger}$ & $3.66[3.61,3.72]$ & $3.67[3.62,3.72]$ & 0.83 \\
\hline & Doctors explained things in an understandable way ${ }^{\dagger}$ & $3.60[3.54,3.66]$ & $3.61[3.56,3.67]$ & 0.73 \\
\hline & Doctor involved you in decisions* & $4.00[3.95,4.06]$ & $3.98[3.93,4.03]$ & 0.49 \\
\hline
\end{tabular}

NOTE: Models adjusted for sex, marital status, severity, age group, ethnicity, length of stay, and emergency room (ER) admission; $95 \%$ confidence intervals (Cl) in brackets. Abbreviations: PCP, primary care physician. * 5 -Point rating scale. ${ }^{\dagger} 4$-Point rating scale.

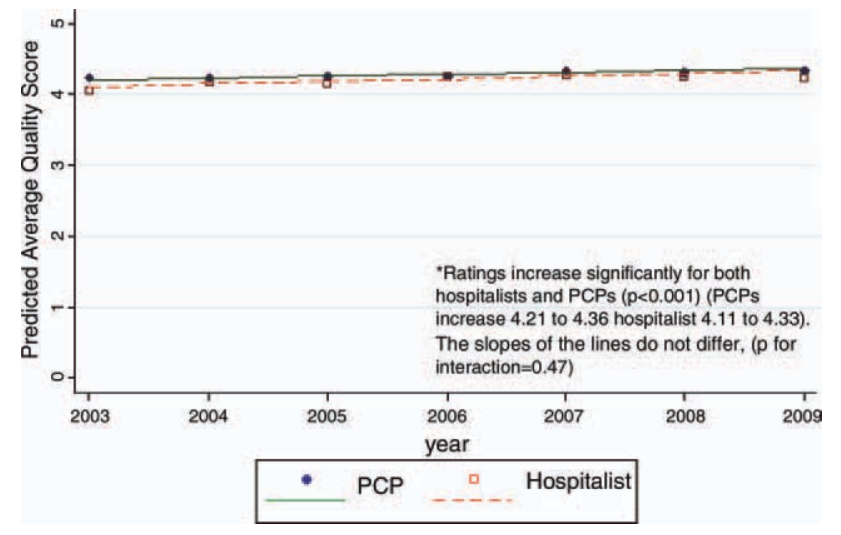

FIG. 2. Trend in quality ratings over time by physician category. Abbreviations: PCP, primary care physician. [Color figure can be viewed in the online issue, which is available at wileyonlinelibrary.com.]

satisfaction with both hospitalists and PCPs showed equivalent rates of improvement over time (Figure 2).

\section{DISCUSSION}

In this observational study of over 8200 patients cared for over 6 years by 347 physicians at 3 hospitals, we found that patient satisfaction with inpatient care provided by hospitalists and primary care doctors was almost identical. As we hypothesized, overall satisfaction with physician care quality, our primary outcome, was slightly greater with primary care doctors; however, the observed difference, 0.04 on a scale of 1 to 5 , cannot be considered clinically significant. All patients were generally satisfied (4.2-4.3 rating on 5point scale) with their inpatient care, and satisfaction scores increased over time. We also found no differences among the specific domains of satisfaction, including communication skills, pain control, and physician behavior. Finally, we found no significant difference in patient satisfaction with physician care quality among the different hospitalist services.

Previous studies of patient satisfaction conducted in the outpatient setting found that continuity of care was an important determinant of trust and, consequently, overall satisfaction. ${ }^{15,16,19,20,22}$ Because hos- pitalist models introduce discontinuity, they might be expected to undermine satisfaction. Surprisingly, few studies have addressed this issue. In a review of the hospitalist studies through 2002, Wachter and Goldman found 19 studies, 5 of which measured patient satisfaction. ${ }^{23}$ Three of these were conducted on teaching services and compared designated faculty hospitalists to traditional ward attendings, who rotated onto the inpatient services 1 to 2 months per year. Primary care doctors were excluded. ${ }^{24-26}$ A fourth study provided a descriptive narrative of the development of the first hospitalist program in Minneapolis, Minnesota, and anecdotally noted no difference in patient satisfaction between the hospitalist and traditional model, but presented no data because the satisfaction "surveys were not designed with publication in mind." 27 The only study to actually assess whether patient satisfaction was greater with hospitalists or PCPs was an observational study by Davis et al., conducted in 1 rural hospital during the first year of its hospitalist program. In that study, 2 hospitalists were compared to 17 PCPs, and patient satisfaction surveys were available for approximately 44 patients managed by hospitalists and 168 patients managed by PCPs. Specific data were not reported, but it was noted that there was no statistical difference in satisfaction between those cared for by hospitalists versus PCPs. ${ }^{28}$ On the basis of these studies, Wachter and Goldman concluded that "surveys of patients who were cared for by hospitalists show high levels of satisfaction, no lower than that of similar patients cared for by their own primary physicians." 23 Wachter and Goldman's review has been highly cited, and we could find no subsequent studies addressing this issue. Our study provides the first real evidence to support this conclusion, including data from 59 hospitalists practicing in 5 separate hospitalist programs at 3 different hospitals.

Our finding that hospitalists maintain satisfaction despite a lack of continuity suggests that other aspects of care may be more important to patient satisfaction. Larson et al. found that physician ability to meet patient's information needs was positively associated 
with patient satisfaction. ${ }^{29}$ Similarly, Tarrant et al. found that patient's trust in a physician improved with increasing communication, interpersonal care, and knowledge of the patient. Interestingly, continuity, ie. the proportion of visits to the usual general practitioner (GP) or duration with the practice, did not correlate with trust. ${ }^{30}$ Finally, a systematic review of determinants of outpatient satisfaction found that continuity has a variable effect on satisfaction. Subjective continuity measures, such as whether patients saw their regular physician on the day they were surveyed, were consistently associated with patient satisfaction, however, quantitative measures including relationship duration were not. ${ }^{31}$

It is also possible that patients believe they value continuity more than they actually do. In 1 survey of inpatients with an established PCP yet cared for by a hospitalist, most agreed that patients receive better care and have more trust in physicians with whom they have long-term relationships. Yet most also had positive opinions of their hospital care. ${ }^{32}$ Similarly, in a survey of over 2500 outpatients, $92 \%$ rated continuity as very important or important, but the majority was unwilling to expend substantial personal time $(88 \%)$, defined as driving greater than 60 minutes, or money $(82 \%)$, defined as spending an additional $\$ 20$ to $\$ 40$ a month, to maintain continuity with their PCP. ${ }^{33}$ Our study appears to confirm the lack of connection between continuity and satisfaction. Even those patients who valued continuity, as evidenced by having an established PCP, were as satisfied with hospitalist physician care as patients who had no established PCP.

Our study has several limitations. First, we report on outcomes of 3 institutions within a single healthcare system, within a limited geographic area. Although our sample included a wide range of patient demographics, hundreds of physicians, and multiple hospitalist models, it is possible that some hospitalist models may provide greater or lesser satisfaction than those we observed. Second, our study was observational, and thus subject to selection bias and confounding. Patients cared for by the hospitalists differed in a number of ways from those cared for by PCPs. We controlled for identifiable confounders such as illness severity, self-perceived health, and admission through the emergency department, but the possibility exists that additional unidentified factors could have affected our results. It is possible other drivers of patient satisfaction, such as amenities, nursing, or food, could have influenced our findings. However, this is unlikely because all patient groups shared these components of hospital experience equally. Third, only a minority of patients could be reached for interview. This is typical for post-hospitalization surveys, and our response rate of $40 \%$ for HCAHPS patients compared favorably to the 2010 HCAHPS national average of $33 \% .{ }^{34}$ Still, the responses of those who could not be reached may have differed from those who were interviewed. Fourth, we identified hospitalists and PCPs by the attending of record, but we were unable to tell who provided care to the patient on any given day. Thus, we could not determine to what extent patients cared for by PCPs were actually seen by their own doctor, as opposed to an associated physician within the practice. Nevertheless, our results are representative of the care model provided by PCPs in the hospital. Similarly, we could not know or compare the number of different attending physicians each patient experienced during their hospitalization. Higher turnover of inpatient physicians may have affected patient satisfaction scores independent of attending physician designation. These are potentially important measures of relationship duration, yet whether duration affects patient satisfaction remains undecided. ${ }^{16-18,20,28,30,32,33}$ We assessed satisfaction using HCAHPS questions, in order to provide objective and meaningful comparisons across hospitals. The HCAHPS instrument, however, is intended to assess patient satisfaction with doctors in general, not with subgroups or individuals, and responses in our study were uniformly high. A more sensitive survey instrument may have yielded different results. Finally, it is possible that individual physicians may possess lower satisfaction scores than others, making the results not representative of hospitalist models as much as specific doctors' care quality. We think this is unlikely since surveys reached over 8000 patients, over 6 years, representing the care of 347 individual physicians. However, hospital medicine is a rapidly evolving field with many divergent organizational structures, and patient satisfaction is bound to fluctuate while there exists high variability in how care is provided.

Over the past decade, the hospitalist model has become one of the dominant models for care of medical inpatients. Compared to the traditional model in which PCPs provide inpatient care, the hospitalist model has a number of advantages, including continuous on-site coverage for increasingly acute patients, specialization, and incentives aligned with the hospital to provide efficient, high-quality care. One concern that remains, however, is that patients may not trust doctors they first meet in the hospital or may be dissatisfied with the lack of continuity from day to day. Our findings are reassuring in this regard. Although patients cared for by hospitalists were slightly less satisfied, the differences could not be considered clinically meaningful and should be outweighed by gains in quality and efficiency. Furthermore, hospitalists can expect to fare well under value-based purchasing. Given the rapid ascension of hospital medicine programs, prospective comparisons of hospitalists and PCPs may no longer be feasible. Future research might employ survey instruments designed specifically to measure patient experience under hospitalist care in 
order to identify methods to maximize patient satisfaction within the hospitalist model.

\section{Acknowledgements}

Jane Garb, MS, Academic Affairs, Baystate Medical Center, contributed to the initial database management and statistical analysis. She received no financial compensation. Dr Adrianne Seiler has received written permission for acknowledgement from Ms Garb.

Dr Adrianne Seiler made substantial contributions to our manuscript's conception and design, data acquisition, analysis, and interpretation, manuscript drafting and critical revision, and administrative support. Dr Paul Visintainer made substantial contributions to our manuscript's data analysis and interpretation, manuscript critical revision, and statistical analysis. Michael Ehresman and Richard Brzostek made substantial contributions to our manuscript's data acquisition, manuscript critical revision, and administrative support. Dr Evan Benjamin made substantial contributions to our manuscript's conception and design, analysis and interpretation of data, manuscript drafting, and administrative support. Dr Winthrop Whitcomb made substantial contributions to our manuscript's data analysis and interpretation, and manuscript critical revision. Dr Michael Rothberg made substantial contributions to our manuscript's conception and design, data analysis and interpretation, manuscript critical revision, and supervision.

Disclosure: Adrianne Seiler, MD, certifies that all coauthors have seen and agree with the contents of the manuscript. Paul Visintainer, $\mathrm{PhD}$, had full access to all of the data in the study and takes responsibility for the integrity of the data and the accuracy of the data analysis. Dr Seiler certifies that none of the authors involved in the study had any potential conflicts of interest nor financial interests. Our study had no funding or sponsorship.

\section{References}

1. American Hospital Association Annual Survey Database. Fiscal Year 2009.

2. Lindenauer PK, Chehabeddine R, Pekow P, Fitzgerald J, Benjamin EM. Quality of care for patients hospitalized with heart failure: assessing the impact of hospitalists. Arch Intern Med. 2002;162: 1251-1256.

3. Lindenauer PK, Rothberg MB, Pekow PS, Kenwood C, Benjamin EM, Auerbach AD. Outcomes of care by hospitalists, general internists, and family physicians. N Engl J Med. 2007;357:2589-2600.

4. Rifkin WD, Burger A, Holmboe ES, Sturdevant B. Comparison of hospitalists and nonhospitalists regarding core measures of pneumonia care. Am J Manag Care. 2007;13:129-132.

5. Rifkin WD, Conner D, Silver A, Eichorn A. Comparison of processes and outcomes of pneumonia care between hospitalists and community-based primary care physicians. Mayo Clin Proc. 2002;77: 1053-1058.

6. Rifkin WD, Holmboe E, Scherer H, Sierra H. Comparison of hospitalists and nonhospitalists in inpatient length of stay adjusting for patient and physician characteristics. J Gen Intern Med. 2004;19: 1127-1132.

7. Roytman MM, Thomas SM, Jiang CS. Comparison of practice patterns of hospitalists and community physicians in the care of patients with congestive heart failure. J Hosp Med. 2008;3:35-41.

8. Meltzer D, Manning WG, Morrison J, et al. Effects of physician experience on costs and outcomes on an academic general medicine service: results of a trial of hospitalists. Ann Intern Med. 2002;137: 866-874.

9. Hackner D, Tu G, Braunstein GD, Ault M, Weingarten S, Mohsenifar $Z$. The value of a hospitalist service: efficient care for the aging population? Chest. 2001;119:580-589.

10. Everett GD, Anton MP, Jackson BK, Swigert C, Uddin N. Comparison of hospital costs and length of stay associated with general intern- ists and hospitalist physicians at a community hospital. Am J Manag Care. 2004;10:626-630.

11. Southern WN, Berger MA, Bellin EY, Hailpern SM, Arnsten JH. Hospitalist care and length of stay in patients requiring complex discharge planning and close clinical monitoring. Arch Intern Med. 2007;167:1869-1874.

12. Coffman J, Rundall TG. The impact of hospitalists on the cost and quality of inpatient care in the United States: a research synthesis. Med Care Res Rev. 2005;62(4):379-406.

13. US Department of Health and Human Services Medicare Hospital Value-Based Purchasing Plan Development Issues Paper. 1st Public Listening Session January 17, 2007. Available at: https://www.cms. gov/AcuteInpatientPPS/downloads/hospital_VBP_plan_issues_paper. pdf. Accessed on May 26, 2011.

14. Hospital Value-Based Purchasing: Measure Explanations. Available at: http://www.healthcare.gov/news/factsheets/valuebasedpurchasing 04292011b.html. Accessed on May 26, 2011.

15. Safran DG, Taira DA, Rogers WH, Kosinski M, Ware JE, Tarlov AR. Linking primary care performance to outcomes of care. J Fam Pract. 1998;47:213-220.

16. Saultz JW, Albedaiwi W. Interpersonal continuity of care and patient satisfaction: a critical review. Ann Fam Med. 2004;2:445-451.

17. Cabana MD, Jee SH. Does continuity of care improve patient outcomes? J Fam Pract. 2004;53:974-980.

18. Fan VS, Burman M, McDonell MB, Fihn SD. Continuity of care and other determinants of patient satisfaction with primary care. J Gen Intern Med. 2005;20:226-233.

19. Mainous AG, Baker R, Love MM, Gray DP, Gill JM. Continuity of care and trust in one's physician: evidence from primary care in the United States and the United Kingdom. Fam Med. 2001;33:22-27.

20. Kao AC, Green DC, Davis NA, Koplan JP, Cleary PD. Patients' trust in their physicians: effects of choice, continuity, and payment method. J Gen Intern Med. 1998;13:681-686.

21. Barros AJ, Hirakata VN. Alternatives for logistic regression in crosssectional studies: an empirical comparison of models that directly estimate the prevalence ratio. BMC Med Res Methodol. 2003;3:21.

22. Wasson JH, Sauvigne AE, Mogielnicki RP, et al. Continuity of outpatient medical care in elderly men. A randomized trial. JAMA. 1984; 252:2413-2417.

23. Wachter RM, Goldman L. The hospitalist movement 5 years later. JAMA. 2002;287:487-494.

24. Palmer HC, Armistead NS, Elnicki DM, et al. The effect of a hospitalist service with nurse discharge planner on patient care in an academic teaching hospital. Am J Med. 2001;111(8):627-632.

25. Meltzer DO, Shah MN, Morrison J, et al. Decreased length of stay, costs and mortality in a randomized trial of academic hospitalists. J Gen Intern Med. 2001;16(suppl):S208.

26. Wachter RM, Katz P, Showstack J, Bindman AB, Goldman L. Reorganizing an academic medical service: impact on cost, quality, patient satisfaction, and education. JAMA. 1998;279(19):1560-1565.

27. Freese RB. The Park Nicollet experience in establishing a hospitalist system. Ann Intern Med. 1999;130:350-354.

28. Davis KM, Koch KE, Harvey JK, Wilson R, Englert J, Gerard PD. Effects of hospitalists on cost, outcomes, and patient satisfaction in a rural health system. Am J Med. 2000;108:621-626.

29. Larson CO, Nelson EC, Gustafson D, Batalden PB. The relationship between meeting patients' information needs and their satisfaction with hospital care and general health status outcomes. Int J Qual Health Care. 1996;8:447-456.

30. Tarrant C, Stokes T, Baker R. Factors associated with patients' trust in their general practitioner: a cross-sectional survey. Br J Gen Pract. 2003;53:798-800.

31. Adler R, Vasiliadis A, Bickell N. The relationship between continuity and patient satisfaction: a systematic review. Fam Pract. 2010;27: 171-178.

32. Hruby M, Pantilat SZ, Lo B. How do patients view the role of the primary care physician in inpatient care? Dis Mon. 2002;48:230-238.

33. Pereira AG, Pearson SD. Patient attitudes toward continuity of care. Arch Intern Med. 2003;163:909-912.

34. Summary of HCAHPS Survey Results. Available at: http://www. hcahpsonline.org/files/12-13-10_Summary_of_HCAHPS_Survey_ Results_December_2010.pdf. Accessed on May 27, 2011. 